\title{
Stability phase diagram of perpendicular magnetic tunnel junction in non-collinear geometry
}

\author{
N. Strelkov, ${ }^{1,2,3,4, *}$ A. Timopheev, ${ }^{1,2,3}$ R. C. Sousa, ${ }^{1,2,3}$ \\ M. Chshiev, ${ }^{1,2,3}$ L. D. Buda-Prejbeanu, ${ }^{1,2,3}$ and B. Dieny ${ }^{1,2,3, \dagger}$ \\ ${ }^{1}$ Univ. Grenoble Alpes, F-38000 Grenoble, France \\ ${ }^{2}$ CEA, INAC-SPINTEC, F-38000 Grenoble, France \\ ${ }^{3}$ CNRS, SPINTEC, F-38000 Grenoble, France \\ ${ }^{4}$ Department of Physics, Moscow Lomonosov State University, Moscow 119991, Russia
}

\begin{abstract}
Experimental measurements performed on $\mathrm{MgO}$-based perpendicular magnetic tunnel junctions show a strong dependence of the stability voltage-field diagrams as a function of the direction of the magnetic field with respect to the plane of the sample. When the magnetic field is applied inplane, systematic nonlinear phase boundaries are observed for various lateral sizes. The simulation results based on the phenomenological Landau-Lifshitz-Gilbert equation including the in-plane and out-of-plane spin transfer torques are consistent with the measurements if a second order anisotropy contribution is considered. Furthermore, performing the stability analysis in linear approximation allowed us to analytically extract the critical switching voltage at zero temperature in presence of an in-plane field. This study indicates that in the non-collinear geometry investigations are suitable to detect the presence of second order term in the anisotropy. Such higher order anisotropy term can yield an easy-cone anisotropy which reduces the thermal stability factor but allows for more reproducible spin transfer torque switching due to a reduced stochasticity of the switching. As a result, the energy per write event decreases much faster than the thermal stability factor as the second order anisotropy becomes more negative. Easy-cone anisotropy can be useful for fast switching STT-MRAM provided the thermal stability can be maintained above the required value for a given memory specification.
\end{abstract}

Keywords: Spintronics, MRAM, STT switching, phase diagrams, non-collinear geometry, LLG linearization

\section{INTRODUCTION}

Magnetic tunnel junctions (MTJ) based on perpendicular magnetic anisotropy (PMA) systems are intensively studied for the development of the next generations of spin transfer torque magnetic random access memories (STT-MRAM). The information is stored by the orientation of the magnetization of the free (storage) layer relatively to that of the reference layer. The readout is performed by measuring the pillar resistance which varies depending on the magnetic configuration due to the MTJ tunnel magnetoresistance. Several key parameters are of importance. First, stability against disturbances, i.e. thermal fluctuations and spurious magnetic fields, usually characterized by the thermal stability factor $\Delta$. Second, the critical current for writing $I_{c 0}$, extrapolated at $1 \mathrm{~ns}$ from the variation of $I_{c}$ versus logarithm of pulse width measured in the thermally activated regime, and finally, the corresponding write voltage which directly influences the write endurance. In the past few years, a lot of $\mathrm{R} \& \mathrm{D}$ efforts were devoted to material developments aiming at the control and optimization of the PMA arising from the magnetic metal/oxide interfaces [1-6] since the use of materials with strong interfacial perpendicular anisotropy allows combining good thermal stability down to quite small lateral sizes (below $30 \mathrm{~nm}$ ) together with relatively low Gilbert damping [7, 8]. Out-of-plane magnetized MgO-based MTJs were identified to be very promising candidates exhibiting magnetoresistance val- ues close to or above $200 \%$, which is required to reduce read access time and read error rates. Writing voltage of about $0.75 \mathrm{~V}$ at $10 \mathrm{~ns}$ pulse width allowing to reach $10^{-6}$ bit error rate were obtained on $11 \mathrm{~nm}$ diameter MTJ pillars [9]. In STT-MRAM, the reversal between the two stable states is enabled by spin transfer torque (STT) $[10,11]$. In the macrospin picture of conventional STT-MRAM, the magnetizations of the free layer and reference layer are parallel or antiparallel to each other at equilibrium. This makes the STT switching inherently stochastic since a large enough thermal fluctuation is required to trigger the magnetization reversal. As a result, higher voltage or longer write pulses are needed to reach sufficiently low bit error rates [12]. Reducing the write current remains one of the most important requirement to achieve sub-10 ns and quasi infinitely enduring STT-MRAM suitable for static random access memory (SRAM) type applications. Introducing non-collinearity between the reference layer magnetization and that of the storage layer is a way to reduce or suppress the stochasticity of the switching. A geometry which received lot of attention is that of orthogonal polarizer and storage layer (so-called OST-MRAM) in which sub-ns switching was demonstrated [13-15]. Recently, it was shown also that non-collinearity between the free layer and the reference layer could be induced by introducing an easy-cone anisotropy in one of the MTJ magnetic electrode, preferably the storage layer [16-18]. This easy-cone anisotropy results from higher order anisotropy term which may 
themselves originate from spatial fluctuations of first order anisotropy [19]. This canted equilibrium state is advantageous for spin torque driven switching since the spin transfer torque becomes immediately effective at the very onset of the write current pulse which strongly attenuates the stochastic character of the switching.

In general, stability field-voltage diagrams are useful tools to study the mechanisms and the properties of STT induced magnetization reversal. However, these diagrams are usually measured in a collinear geometry, i.e. with the external magnetic field applied parallel to the easy-axis of MTJ magnetic layers (free layer and polarizer) [20, 21]. Considering the rising interest for noncollinear geometries, it is also interesting to study these phase diagrams in such geometry, i.e. in situations where the field is applied at some angle with respect to the normal to the layers, introducing thus a non-collinearity in the static configuration of the storage and polarizer layers. Non-collinear configurations of magnetic electrodes influences the switching characteristics of the storage layer and therefore the field-voltage phase boundaries of the stability diagram.

In this study, the voltage-field stability diagrams were investigated experimentally and by simulations as a function of the applied field orientation from easy-axis (perpendicular to the plane of the layers) to hard-plane (inplane). Experimental measurements were performed on MgO-based magnetic tunnel junctions with various diameters ranging from $50 \mathrm{~nm}$ to $150 \mathrm{~nm}$. We found that the shape of the stability field-voltage diagrams depends strongly on the direction of the applied field while the bistable $\mathrm{P} / \mathrm{AP}$ region preserves its symmetry around the origin.

We have found also a quite noticeable difference in the shape of measured and simulated hard-axis VH diagrams, $\mathrm{H}$ in the hard plane, when considering that the anisotropy is only of uniaxial form. However, a good agreement between experiments and simulations is recovered when a second order anisotropy contribution is introduced in the model. The signature of this higher order anisotropy term is not always visible in the collinear geometry but clearly shows up once the field is applied away from the normal to the plane of the sample. Such second order anisotropy contribution is similar to that reported previously [17, 18, 22-25].

The paper is organized as follows. The first part provides the description of the samples, protocol of measurement and a summary of the experimental results. The phenomenological model used for the simulation is introduced in the second part followed by a discussion on the stability diagrams predicted under different conditions. The analytical model developed for in-plane configuration allows to easily derive the phase boundaries and confirms the role of the second order contribution to the anisotropy on the observed shape of the stability diagrams. The impact of this second order contribution on the magnetic thermal stability and on the STT efficiency of the magnetization switching is analyzed at the end from write energy point of view.

\section{EXPERIMENTAL STABILITY DIAGRAMS}

Perpendicular MTJ (pMTJ) pillars arrays with nominal diameters ranging between $50 \mathrm{~nm}$ and $150 \mathrm{~nm}$ were fabricated from a MTJ stack grown by DC and RF magnetron sputtering on a thermally oxidized Si substrate. The stack has a bottom reference pMTJ structure with the polarizer layer fixed by a synthetic antiferromagnet. The free layer is sandwiched between two MgO layers. Saturation magnetization of the free layer was measured to be $1030 \mathrm{kA} / \mathrm{m}$. Current in-plane magnetotransport

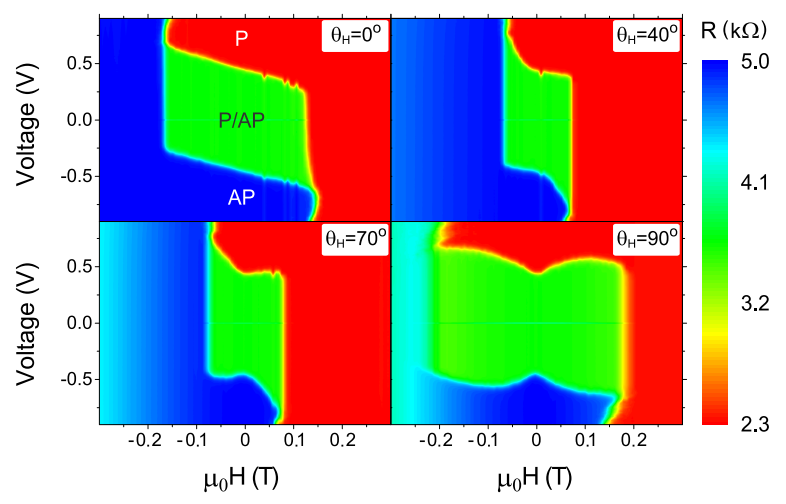

FIG. 1. Experimental stability VH diagrams of $80 \mathrm{~nm}$ diameter MTJ at room temperature for $\theta_{H}=0^{\circ}, 40^{\circ}, 70^{\circ}$ and $90^{\circ}$. Voltage pulse length was $100 \mathrm{~ns}$. The color scale is related to the resistance of MTJ.

measurements yielded $\mathrm{RA}=5.7 \Omega \cdot \mu \mathrm{m}^{2}$ and $\mathrm{TMR}=126 \%$. A second $\mathrm{MgO}$ barrier was introduced on top of the storage layer to increase the perpendicular anisotropy of the free layer. Its resistance-area (RA) product is much lower than that of the main tunnel barrier. Additional information on these samples can be found in Ref. [18]. The procedure for $\mathrm{VH}$ stability diagram measurements is similar to that described in Ref. [20]. A single pMTJ pillar was wire bonded and placed in a physical properties measurement system (PPMS) on a sample rotator. In this work, instead of measuring the device resistance-magnetic field (R-H) loops at constant writing pulse amplitude, as in Ref. [20], we measured a device resistance-writing pulse voltage $(\mathrm{R}-\mathrm{V})$ loops at each magnetic field point. Within each R-V loop, a sequence of writing pulses with continuously changing amplitude was applied and after each writing pulse the resistance of the pMTJ pillar was measured by applying a small bias current $(\sim 1 \mu \mathrm{A})$. Considering the stochasticity of the switching, at each $H$ point, the measurement of an $\mathrm{R}-\mathrm{V}$ loop was repeated 30 times 
and then averaged. For a magnetic field applied along the easy axis, i.e. in the out-of-plane direction, a final diagram is constructed from $\mathrm{R}-\mathrm{V}$ loops at different $\mathrm{H}$ field points, as in FIG. 1 for $\theta_{H}=0^{\circ}$. It shows three main resistance states: high resistance region, where only the antiparallel (AP) configuration is stable, low resistance region, with only the parallel $(\mathrm{P})$ state being stable, and bistable $\mathrm{P} / \mathrm{AP}$ region with the resistance equal to the half sum of the resistances in $\mathrm{AP}$ and $\mathrm{P}$ states for a given $H$ field point. In case of non-collinear configuration of the magnetic electrodes, introduced here by the tilted magnetic field, high and low resistance values become fielddependent, so that color gradients representing variable resistance states appear in all three regions (see, for example FIG. $1 \theta_{H}=90^{\circ}$ ).

Upon varying the orientation of the magnetic field, the shape of the VH diagram is evolving. For the easy-axis case $\left(\theta_{H}=0^{\circ}\right)$, the $\mathrm{VH}$ stability diagram exhibits the parallelogram shape already reported in Ref. [26]: two vertical field-driven phase boundaries corresponding to the coercive fields (positive and negative) and two parallel voltage-driven phase boundaries with linear voltagefield variation corresponding to the STT induced magnetization switching. When increasing the field angle with respect to the normal to the layers, the coercive field decreases up to $45^{\circ}$ and then increases back for higher angles. This variation follows the common variation of the Stoner-Wohlfarth model [27]. However, the voltage-driven phase boundaries are not following a linear VH variation, developing a flying seagull shape once the magnetic field is applied close to the hard-plane and the switching voltage always increases with increasing applied in-plane field modulus. Nevertheless, the bistable region still keeps a rough central symmetry whatever the magnetic field angle. In FIG. 1, one may note however a more pronounced resistance variation at large negative fields than at large positive fields for the measurements performed with field applied at $70^{\circ}$ or $90^{\circ}$. This can be ascribed to a slight dipolar field exerted by the reference layer on the storage layer. This dipolar coupling is visible on the stability diagram measured at $0^{\circ}$ from the shift of the bistable region along the field axis (see supplemental material).

The experimental investigation was carried out systematically for pillars with diameters ranging between $50 \mathrm{~nm}$ and $150 \mathrm{~nm}$. The evolution of the stability diagrams with the orientation of the external field was found to be similar to that reported in FIG. 1 whatever the lateral size and it is qualitatively the same at lower temperatures (at least for $T<190 \mathrm{~K}$ ). Some shifts of the boundaries are observed but without changing the shape of the curves. This behavior seems to be generated by a mechanism independent of the size of the sample and most probably intrinsic to the pillar pMTJ stack structure.

In order to obtain a better understanding on the experimental results, a phenomenological macrospin model based on the Landau-Lifshitz-Gilbert equation was used. It is described in the next section.

\section{NUMERICAL STABILITY DIAGRAMS}

The response of the magnetization of the free layer to the simultaneous action of the static applied field and spin transfer torques due to the injection of a voltage pulse was simulated using the Landau-Lifshitz-Gilbert equation (LLG) $[28,29]$ including two additional terms related to the STT (damping-like and field-like). The phenomenological equation of the magnetization dynamics is written as:

$$
\begin{aligned}
\frac{d \mathbf{m}}{d t}= & -\gamma\left(\mathbf{m} \times \mu_{0} \mathbf{H}_{\mathrm{eff}}\right)+\alpha\left(\mathbf{m} \times \frac{d \mathbf{m}}{d t}\right) \\
& -\gamma a_{\|} V[\mathbf{m} \times(\mathbf{m} \times \mathbf{p})]+\gamma a_{\perp} V^{2}(\mathbf{m} \times \mathbf{p}),
\end{aligned}
$$

where $\mathbf{m}$ is a unit vector along the free layer magnetization, $\alpha$ is the Gilbert damping constant, $\gamma$ is the gyromagnetic ratio of free electron, $\mu_{0}$ is the vacuum permeability, $\mathbf{p}$ is a unit vector along the current spin polarization. The damping-like STT torque is supposed to

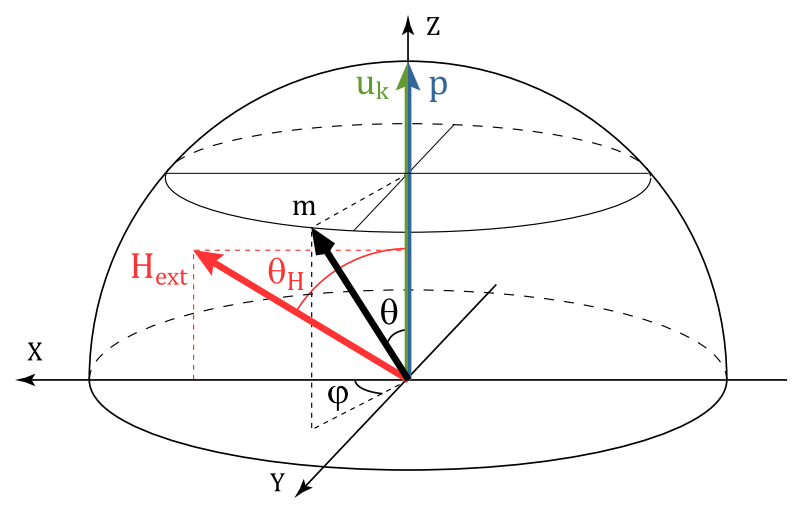

FIG. 2. Geometry of the non-collinear MTJ system. OZ is along the uniaxial anisotropy axis $\mathbf{u}_{\mathbf{K}}$ (perpendicular to the MTJ $\mathrm{x}$-y plane), $\mathbf{m}$ - free layer magnetization, $\theta$ - angle between magnetization and $\mathbf{z}$-axis, $\mathbf{p}$ - unit vector along current spin polarization, $\mathbf{H}_{\text {ext }}-$ external applied field and $\theta_{H}-$ angle between applied field and z-axis.

vary linearly with the applied voltage $\mathrm{V}$ while the fieldlike STT torque has a quadratic voltage dependence in case of symmetric MTJs. The phenomenological transport parameters $a_{\|}$and $a_{\perp}$ can be evaluated from freeelectron, tight-binding or first principles [30-38] models of the MTJ. The effective field $\mathbf{H}_{\text {eff }}$ is derived from the Gibbs free energy density functional which in macrospin approximation reads:

$$
\begin{aligned}
E= & -K_{1}\left(\mathbf{u}_{\mathbf{K}} \cdot \mathbf{m}\right)^{2}-\mu_{0} M_{s} \mathbf{H}_{\mathrm{ext}} \cdot \mathbf{m} \\
& +\frac{1}{2} \mu_{0} M_{s}^{2}\left(N_{x x} m_{x}^{2}+N_{y y} m_{y}^{2}+N_{z z} m_{z}^{2}\right),
\end{aligned}
$$


where $\mathbf{H}_{\text {ext }}$ is the external magnetic field, $\mathbf{u}_{\mathbf{K}}$ is the magnetocrystalline axis, $K_{1}$ is the first order uniaxial anisotropy constant $\left(K_{1}>0\right), M_{s}$ is the saturation magnetization of free layer and $\left(N_{x x}, N_{y y}, N_{z z}\right)$ the diagonal terms of the demagnetizing tensor assuming the sample can be approximated with an ellipsoidal shape.

The geometry of the non-collinear pMTJ system is shown in FIG. 2. The uniaxial anisotropy is directed along the $\mathrm{z}$-axis $\mathbf{u}_{\mathbf{K}}=(0,0,1)$, the external magnetic field $\mathbf{H}_{\text {ext }}$ is in the xz-plane tilted away from z-axis with the polar angle $\theta_{H}$ and the current spin polarization points along the $\mathrm{z}$-axis $\mathbf{p}=(0,0,1)$.

To compute the numerical VH diagrams, a procedure similar to the experimental one is followed. The free layer magnetization is relaxed under a given applied field and a writing pulse is applied for $100 \mathrm{~ns}$. The state of the free layer is measured after twice the pulse length. The amplitude of the voltage is gradually changed in the loop in the following sequence $0 \rightarrow V_{\max } \rightarrow V_{\min } \rightarrow 0$. The LLG solver uses time steps shorter than 2 fs. The average value of the $m_{z}$ component of magnetization for a given voltage in the loop is plotted as a point on the diagram.
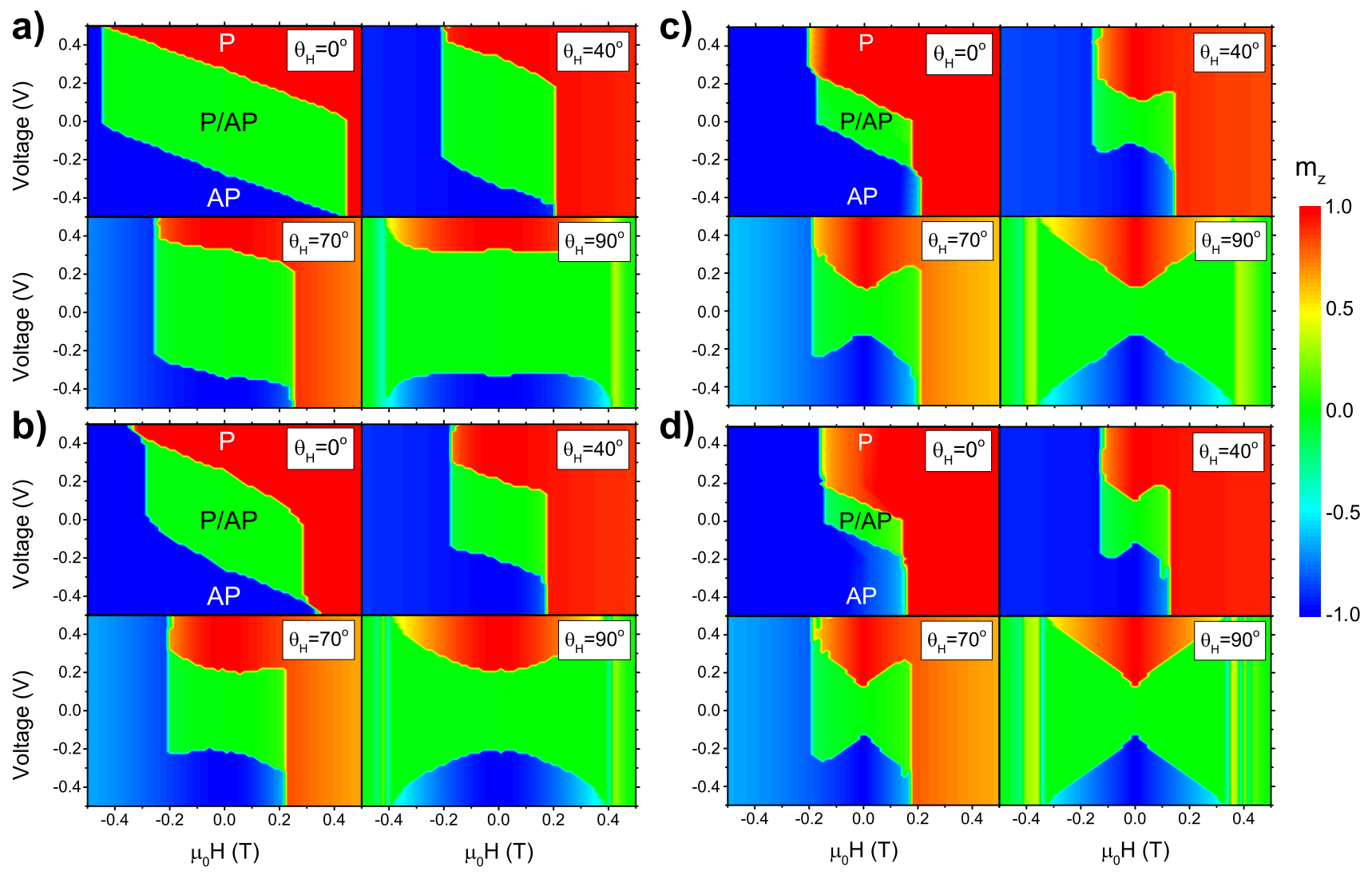

FIG. 3. Calculated stability diagrams at $T=0 \mathrm{~K}$ with field applied at $0^{\circ}, 40^{\circ}, 70^{\circ}$ and $90^{\circ}$ away from the z-axis. Simulation parameters: $K_{1}=778 \mathrm{~kJ} / \mathrm{m}^{3}, M_{s}=10^{6} \mathrm{~A} / \mathrm{m}, N_{x x}=N_{y y}=0.04, N_{z z}=0.92, \alpha=0.01, a_{\|}=16 \cdot 10^{-3} \mathrm{~T} / \mathrm{V}, a_{\perp}=10^{-3} \mathrm{~T} / \mathrm{V}^{2}$, a second order term of the form $-K_{2} \cos ^{4} \theta$ in the anisotropy a) $K_{2}=0 \mathrm{~J} / \mathrm{m}^{3}$, b) $K_{2}=-50 \mathrm{~kJ} / \mathrm{m}^{3}$, c) $K_{2}=-100 \mathrm{~kJ} / \mathrm{m}^{3}$ and d) $K_{2}=-$ $150 \mathrm{~kJ} / \mathrm{m}^{3}$.

The VH stability diagrams were computed for several orientations of the applied field and are shown in FIG. 3a. For the collinear case $\left(\theta_{H}=0^{\circ}\right)$, the numerical and experimental diagrams are very similar in shape. The switching $V(H)$ boundaries are linear and parallel for the $\mathrm{P}$ to AP and AP to P transitions. Varying the polar angle $\theta_{H}$ gradually changes the shape of the stability diagrams. The slope of the $V(H)$ switching boundaries gradually decreases and these boundaries acquire some curvature. At $\theta_{H}=90^{\circ}$ the switching boundaries become flat over a large range of field around zero field. However, close to the in-plane saturation field, the switching volt- age increases sharply.

Changing the amplitude of the anisotropy constant shifts the phase boundaries but the shape of the diagrams remains the same. At this point, the numerical investigation is in disagreement with the experimental observations. Full $3 \mathrm{D}$ micromagnetic simulations, not shown here, were also carried out and the results were similar to the present macrospin results. Therefore, the shape of the experimental diagrams could not be explained under the present model assumptions even by taking into account possible non-uniform magnetization dynamics.

Several recent publications have reported the possible 
presence of an additional second order anisotropy term of the form $-K_{2} \cos ^{2} \theta[18,22]$ in structures similar to ours. Under this assumption, the anisotropy contribution to the free energy density (2) is modified as follows:

$$
E_{a}=-K_{1}\left(\mathbf{u}_{\mathbf{K}} \cdot \mathbf{m}\right)^{2}-K_{2}\left(\mathbf{u}_{\mathbf{K}} \cdot \mathbf{m}\right)^{4} .
$$

Uniaxial magnetocrystalline anisotropy in hexagonal crystal along c-axis, for instance, is usually described as a power series of sinuses, $\tilde{K}_{1} \sin ^{2} \theta+\tilde{K}_{2} \sin ^{4} \theta$ (see Eq. (1) in Ref. [24]). In our case, as well as in several other works related to perpendicular anisotropy study in STTMRAM [18, 23, 39, 40], it was found to be more convenient to express the anisotropy as cosines series expansion in Eq. (3). Both notations can be mathematically transformed into one another but the derived constants differ in magnitudes and/or signs. In the first case (sinuses expansion), a continuous increase of $-K_{2} / K_{1}$ ratio with $K_{2}<0$ and $K_{1}>0$ will describe emergence of an easy-cone state from the basal plane with the cone angle shrinking. In contrast, the second notation (cosine expansion) yields emergence of the easy-cone state from the film normal with further cone angle increase. The latter case corresponds to our experimental observations reported in Ref. [18]: upon decreasing the temperature, the easy-cone starts developing from the out-of-plane direction which in the second notation (cosines expansion) gives a continuous increase of $K_{2} / K_{1}$ ratio versus temperature. On a contrary, the first notation would yield a discontinuity of this ratio when the magnetization starts departing from the normal to the plane.

Based on this modified model, FIG. 3(b,c,d) summarize the VH stability diagrams computed with three different values of $K_{2}$. In the collinear case $\theta_{H}=0^{\circ}$, the shape of the diagrams is unchanged. Only the size of the bistable region shrunk. Almost parallel linear switching boundaries are observed as in the case $K_{2}=0$. However, once the symmetry is broken by the field direction, several changes arise. Assuming a stronger second order contribution (i.e. increasing $\left|K_{2}\right|$ ), we find a better correlation with the experimental diagrams.

This systematic numerical study points out the importance of the second order anisotropy term and shows that the collinear configuration alone does not allow to identify its role. In contrast, investigating the switching behavior in non-collinear configuration allows elucidating the presence and role of higher anisotropy terms.

Due to this second order term, when the condition $K_{1}-\mu_{0}\left(N_{z z}-N_{x x}\right) M_{s}^{2} / 2+2 K_{2}<0$ is fulfilled, the magnetization of the magnetic layer is no longer along the normal to the layers at zero field, but rather lies on an easy-cone surface [22]. Both values of $K_{2}$ considered in FIG. 3b,c are not sufficiently negative to allow the onset of the easy-cone state since the $K_{2}$ threshold is $112.7 \mathrm{~kJ} / \mathrm{m}^{3}$. Nevertheless, close to the threshold for the onset of the easy-cone regime, the shape of the diagrams for $\theta_{H}=90^{\circ}$ is significantly affected by the presence of the second order anisotropy term. Therefore, to obtain the "seagull" shape of the VH stability diagram, it is not necessary to be in an established easy-cone regime.

The presence of a second-order anisotropy also explains the difference in slopes $d V / d H$ of the critical lines at positive and negative fields at intermediate angles of application of the field (see the diagram at e.g. $40^{\circ}$ in FIG. 1). If one considers the energy surface defined by the anisotropy and the z-component of the applied field, the application of the field in the positive direction yields a reduction in the easy-cone opening angle. In contrast, if the field is applied in the negative direction, this results in an increase in the easy-cone opening angle. In the former case, the critical voltage for magnetization switching tends to increase whereas in the latter one, it tends to decrease thus explaining the asymmetry seen in FIG. 1 at $40^{\circ}$. Conversely in the AP state, the situation is reversed so that the slope $d V / d H$ is larger at positive fields than at negative fields.

At high fields near the saturation, there is an unstable region in which thermal fluctuations may affect the shape of the stability diagram. To check the impact of the thermal fluctuations, at finite temperature, a fluctuating term corresponding to a Gaussian distributed thermal field $\mathbf{H}_{\mathrm{TR}}$ was added to the total effective field $\mathbf{H}_{\mathrm{eff}}$ in LLG equation (1). This fluctuating field has the following properties [41]:

$$
\begin{aligned}
\left\langle\mathbf{H}_{\mathrm{TR}}(t)\right\rangle & =0, \\
\left\langle\mathbf{H}_{\mathrm{TR}}(t) \mathbf{H}_{\mathrm{TR}}\left(t^{\prime}\right)\right\rangle & =\frac{2 \alpha k_{B} T}{\gamma \mu_{0} M_{s} \Omega} \delta\left(t-t^{\prime}\right),
\end{aligned}
$$

where $k_{B}$ is Boltzmann constant and $\Omega$ is the sample volume. The time integration is based on the Heun predictor corrector scheme [42] using a time step lower than 2 fs. The stability diagrams were recomputed with tempera-

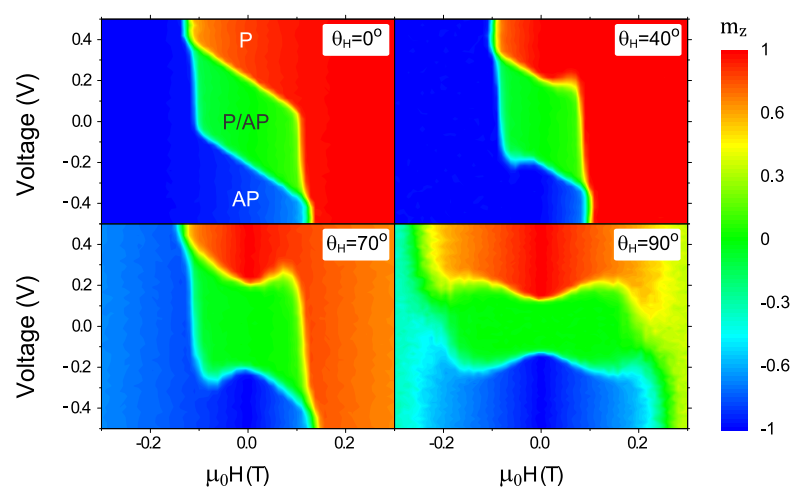

FIG. 4. Numerical stability diagrams at $300 \mathrm{~K}$ with $K_{2}=-$ $150 \mathrm{~kJ} / \mathrm{m}^{3}, \Omega=1.96 \cdot 10^{3} \mathrm{~nm}^{3}, \quad a_{\|}=6 \cdot 10^{-3} \mathrm{~T} / \mathrm{V}$ using 100 events per point. Other parameters are the same as in FIG. 3.

ture, each point being the average of 100 events. The 
updated diagrams are plotted in FIG. 4 for a constant value of $K_{2}=-150 \mathrm{~kJ} / \mathrm{m}^{3}$. One might notice that the temperature leads to smoother boundaries on the diagram at high fields, the similarity with the experimental diagrams is improved and the increase of threshold voltage with applied field remains in the case $\theta_{H}=90^{\circ}$. Again, we find that the non-collinear geometry is required to reveal the influence of the second order anisotropy term.

Regardless of its origin [19], this second order anisotropy contribution has a significant impact on the static and dynamic properties of the magnetic layer and should be considered in the optimization of the pillar stack composition and nanofabrication.

\section{ANALYTICAL SOLUTION FOR IN-PLANE FIELD CONFIGURATION}

The extreme case where the magnetic field is applied in the plane of the free layer $\theta_{H}=90^{\circ}$ is very interesting since the signature of the second order contribution to the anisotropy is most evident in this case. Finding an analytical expression for the critical $V(H)$ switching boundary would allow a straightforward characterization of the sample. Thus we have developed hereafter an analytical approach to predict the behavior of the magnetic free layer with a given set of material parameters when the field is applied in the x-y plane.

The approach comprises two steps. First, the equilibrium angle $\theta_{0}\left(H_{x}\right)$ between the magnetization of the free layer and the normal to the layers plane is found for a given value of the in-plane magnetic field $H_{x}$ with no applied voltage. Second, the LLG equation with spin torque terms Eq. (1) is linearized near that initial equilibrium angle $\theta_{0}\left(H_{x}\right)$ and the stability conditions are derived following a similar procedure to that reported in Ref. [26].

In spherical coordinates, for a continuous thin film $N=$ $(0,0,1)$. Assuming $\theta_{H}=90^{\circ}$ and considering only the damping-like term due to the out-of-plane polarizer $\mathbf{p}=$ $(0,0,1)$, the LLG equation Eq. (1) reads:

$$
\begin{aligned}
\dot{\theta}= & \frac{\gamma}{1+\alpha^{2}}\left(H_{x}(\alpha \cos \theta \cos \varphi-\sin \varphi)\right. \\
& \left.-\sin \theta\left(\alpha \cos \theta \frac{2\left(K+2 K_{2} \cos ^{2} \theta\right)}{\mu_{0} M_{s}}+\frac{a_{\|}}{\mu_{0}} V\right)\right), \\
\dot{\varphi}= & -\frac{1}{\sin \theta} \frac{\gamma}{1+\alpha^{2}}\left(H_{x}(\cos \theta \cos \varphi+\alpha \sin \varphi)\right. \\
& \left.-\sin \theta\left(\cos \theta \frac{2\left(K+2 K_{2} \cos ^{2} \theta\right)}{\mu_{0} M_{s}}-\alpha \frac{a_{\|}}{\mu_{0}} V\right)\right),
\end{aligned}
$$

where $K=K_{1}-\mu_{0} M_{s}^{2} / 2$ is an effective uniaxial anisotropy constant and $H_{x}$ is the x-component of the external field vector $\mathbf{H}_{\mathrm{ext}}=\left(H_{x}, 0,0\right)$.
At equilibrium, the time derivative of the polar angle $\theta$ vanishes and thus the left part of the first equation in Eq. (5) has to be zero. Without applied voltage, since the field is supposed to be along the $\mathrm{x}$-axis, the azimuthal angle $\varphi_{0}=0^{\circ}$ and the problem is reduced to the following cubic equation:

$$
2 K \sin \theta_{0}+4 K_{2}\left(\sin \theta_{0}-\sin ^{3} \theta_{0}\right)-\mu_{0} H_{x} M_{s}=0 .
$$

The equation has three solutions for $\sin \theta_{0}$ but only one is suitable and has a real value. The equilibrium polar angle $\theta_{0}$ is thus uniquely determined. There are two regimes depending on the strength of the $K_{2}$ constant with respect to the effective $K$ and the solution is expressed as follows:

a) if $K+2 K_{2}<0$ :

$$
\sin \theta_{0}\left(H_{x}\right)=2 \sqrt{\frac{1-\varkappa}{3}} \cos \left(\frac{\arccos \xi}{3}\right),
$$

b) if $K+2 K_{2}>0$ :

$$
\sin \theta_{0}\left(H_{x}\right)=2 \sqrt{\frac{1-\varkappa}{3}} \sin \left(\frac{\arcsin \xi}{3}\right),
$$

where $\varkappa=K /\left(2\left|K_{2}\right|\right), E_{H}=\mu_{0} H_{x} M_{s}$ and

$$
\xi=-\frac{27 E_{H}}{8 K_{2}[3(1-\varkappa)]^{3 / 2}} .
$$

In the limit case of zero applied field, the equilibrium polar angle $\theta_{0}$ is given by:

$$
\sin \theta_{0}(0)= \begin{cases}\sqrt{1-\varkappa}, & K+2 K_{2}<0 \\ 0, & K+2 K_{2}>0\end{cases}
$$

One might note that the above case a) corresponds to the situation of the existence of an easy-cone state as recently reported in Ref. [43] while the case b) is the common perpendicular state ("up" or "down").

The relations given by Eqs. (7) and (8) are used to find the equilibrium value $m_{x}=\sin \theta_{0}$ upon varying the $H_{x}$ field. The corresponding magnetization curves are plotted in FIG. 5 for several values of $K_{2}$.

Once the equilibrium magnetization is found without any applied voltage and fixed in-plane field, one might proceed to the linearization of the system of equations (5) with the following substitution of variables: $\theta \rightarrow \theta_{0}+\delta \theta$, $\varphi \rightarrow \varphi_{0}+\delta \varphi$ and keeping just the first order terms in the developments. The polar angle $\theta_{0}$ is an equilibrium angle given by Eqs. (7) or (8) depending on the $K_{2}$ constant value while the azimuthal angle $\varphi_{0}$ is found from the first equation in (5) if $\dot{\theta}=0$ under applied voltage. In case of small deviations of $\varphi_{0}$ angle $\cos \varphi_{0} \approx 1$ and:

$$
\sin \varphi_{0}=-\frac{a_{\|}}{\mu_{0} H_{x}} V \sin \theta_{0} .
$$




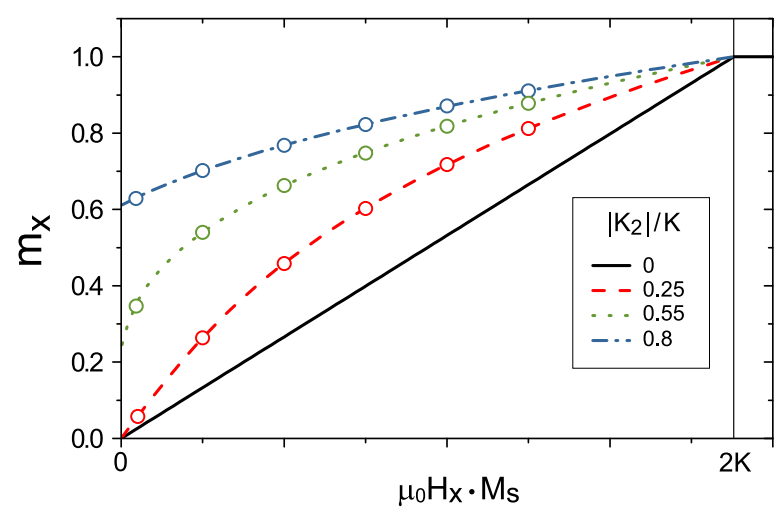

FIG. 5. Dependence of equilibrium $m_{x}=\sin \theta_{0}$ versus $H_{x}$ for different values of $K_{2}$ according to Eq. (7) and Eq. (8). Colored circles are results issued from the LLG integration for the same parameters.

This expression imposes a restriction on some parameters of the system such that the following condition is satisfied:

$$
\left|\frac{a_{\|}}{\mu_{0} H_{x}} V \sin \theta_{0}\right| \ll 1 .
$$

This restriction is intrinsically valid for weak $K_{2}$ case for any value of the $H_{x}$. In contrast, it fails for very small $H_{x}(<1 \mathrm{mT}$, planar saturation field $\sim 300 \mathrm{mT})$ and small voltages in the case for the strong $K_{2}$ since there is no any static equilibrium in such condition as previously reported by Jang et al. [43] for $H_{x}=0$. Using (7) and (8) we can express this condition in the limit $H_{x} \rightarrow 0$ :

$$
\begin{aligned}
a_{\|} V & \ll \lim _{H_{x} \rightarrow 0} \frac{\mu_{0} H_{x}}{\sin \theta_{0}\left(H_{x}\right)}= \\
& = \begin{cases}-\frac{4\left(K+2 K_{2}\right)}{M_{s}}, & K+2 K_{2}<0 \\
\frac{2\left(K+2 K_{2}\right)}{M_{s}}, & K+2 K_{2}>0\end{cases}
\end{aligned}
$$

Relation given by Eq. (13) is valid above and below the threshold but not in the vicinity of $K+2 K_{2}=0$ (i.e. crossover from easy axis to easy-cone). The linearization of the system of equations (5) around the existing equilibrium state at some field $H_{x}$ and applied voltage $V$ allows to deduce the following set of two equations:

$$
\begin{aligned}
\dot{\delta} \theta & =A_{0}+A_{1} \delta \varphi+A_{2} \delta \theta, \\
\dot{\delta} \varphi & =B_{0}+B_{1} \delta \varphi+B_{2} \delta \theta,
\end{aligned}
$$

where the coefficients $A_{i}, B_{i}$ are given in the Appendix. The solution $\delta \theta(t)$ and $\delta \varphi(t)$ of this system of equations contains an exponential time dependence such that:

$$
\begin{aligned}
& \delta \theta(t), \delta \varphi(t) \sim \\
& \exp \left[\frac{1}{2}\left(A_{2}+B_{1} \pm \sqrt{\left(A_{2}-B_{1}\right)^{2}-4 A_{1} B_{2}}\right) t\right]
\end{aligned}
$$

where $\sqrt{\left(A_{2}-B_{1}\right)^{2}-4 A_{1} B_{2}}$ is a term always imaginary, so that the critical line (between increasing and decreasing solutions) is derived from the expression $A_{2}+B_{1}=0$ :

$$
\begin{aligned}
& 2 a_{\|} V_{\text {cr }} \cos \theta_{0}+\alpha \mu_{0} H_{x} \\
& \times\left(\sin \theta_{0}+\frac{1}{\sin \theta_{0}}\right) \sqrt{1-\left(\frac{a_{\|} V_{\text {cr }}}{\mu_{0} H_{x}} \sin \theta_{0}\right)^{2}} \\
& +\frac{2 \alpha}{M_{s}}\left(\left(K+K_{2}\right) \cos 2 \theta_{0}+K_{2} \cos 4 \theta_{0}\right)=0,
\end{aligned}
$$

where $V_{\text {cr }}$ is a critical voltage. It is important to note that this critical voltage corresponds to the voltage at which the solution of the LLG equation becomes unstable indicating that the magnetization starts entering into a precession regime. But this does not mean it is going to switch to the opposite hemisphere and therefore this critical voltage is not the switching voltage. The latter can have slightly larger values than $V_{\text {cr }}$. In the case $a_{\|} V_{\text {cr }} \ll\left(K+2 K_{2}\right) / M_{s}$ and according to Eqs. (12) and (13), expression (16) can be simplified allowing to express $V_{\text {cr }}$ as:

$$
\begin{aligned}
& 2 a_{\|} V_{\mathrm{cr}} \cos \theta_{0}+\alpha \mu_{0} H_{x}\left(\sin \theta_{0}+\frac{1}{\sin \theta_{0}}\right) \\
& +\frac{2 \alpha}{M_{s}}\left(\left(K+K_{2}\right) \cos 2 \theta_{0}+K_{2} \cos 4 \theta_{0}\right)=0,
\end{aligned}
$$

Relation given by Eq. (16) is the major result of our analytical model since it provides the dependence of the critical voltage on the applied field for any set of material parameters. In FIG. 6, the critical voltage given by Eq. (16) is plotted (black dotted-line) for different values of the anisotropy constant $K_{2}$. The analytical results are superposed on the numerical diagrams obtained from the time integration of the full LLG equation (1) with the same parameters. One can note a very good agreement between the analytical model and the numerical model.

FIG. 7(a) shows critical voltage $V_{\text {cr }}$ as extracted from Eq. (16). For a better visualization, only one quadrant of the full VH diagram is shown for weak $K_{2}$ and negative field and strong $K_{2}$ and positive field respectively. The critical lines are evolving progressively with the $K_{2}$ variation, the biggest change is expected close to the threshold.

\section{ENERGETIC EFFICIENCY OF THE MAGNETIZATION SWITCHING}

One important parameter for the application is the estimation of the critical current with no applied field. This is straightforward for our analytical model. Using Eqs. (10) and (17) we can find a critical voltage in the limit of zero field:

$$
V_{\mathrm{cr}, 0}=\left\{\begin{array}{ll}
\frac{2 \alpha}{a_{\|} M_{s}}\left(K+2 K_{2}\right), & K+2 K_{2}>0 \\
0, & K+2 K_{2}<0
\end{array} .\right.
$$




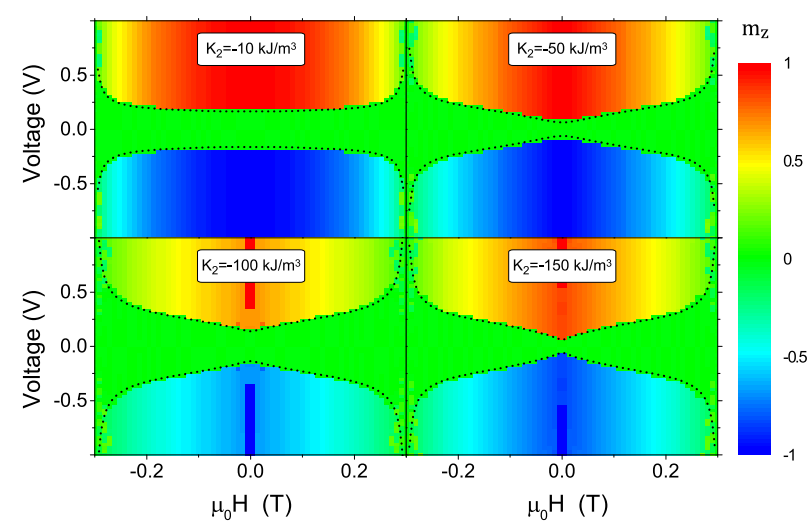

FIG. 6. Comparison of analytical result (black dotted line) with result of numerical simulations. $T=0 \mathrm{~K}, K_{1}=778 \mathrm{~kJ} / \mathrm{m}^{3}$, $K=150 \mathrm{~kJ} / \mathrm{m}^{3}, \alpha=0.01, M_{s}=10^{6} \mathrm{~A} / \mathrm{m}, N_{x x}=N_{y y}=0, N_{z z}=1$, $a_{\|}=16 \cdot 10^{-3} \mathrm{~T} / \mathrm{V}, a_{\perp}=0, K_{2, \mathrm{cr}}=-75 \mathrm{~kJ} / \mathrm{m}^{3}$.

The first expression is similar to the result obtained recently (see Eq. (19) in Ref. [26]) if $K_{2}=0$ and for this case the critical voltage coincides with the switching voltage at zero field $V_{\mathrm{sw}, 0}$. In the easy-cone regime, $V_{\mathrm{cr}, 0}$ equals to zero since once the voltage is applied the magnetization precesses around the easy-cone [43]. For this very symmetric configuration it is possible to estimate the switching voltage using the approach from Ref. [24] and the $V_{\mathrm{sw}, 0}$ reads as following:

$$
V_{\mathrm{sw}, 0}=\frac{\alpha}{a_{\|} M_{s}} \sqrt{\frac{(2 K)^{3}}{27\left|K_{2}\right|}}
$$

The variation of the critical voltage given by Eq. (17) versus $K_{2}$ value is plotted in FIG. 7 (b). If $K+2 K_{2}>0$, the presence of $K_{2}$ allows reducing linearly this critical voltage $V_{\mathrm{cr}, 0}$ upon reinforcing the second order anisotropy, the smallest value being reached close to the easy-axis to easy-cone transition given by $K+2 K_{2}=0$. Once the easy-cone anisotropy starts increasing, the critical voltage increases also. In the case of strong $K_{2}$ and in the limit of very small applied field, one might notice that the analytical lines become shorter and the numerical values are not following anymore the analytical prediction. This discrepancy is due to the fact that before the switching in a very symmetric geometry, the magnetization oscillates on the easy-cone as predicted by [43]. This is an illustration of the difference between $V_{\text {cr }}$ and switching voltage $V_{\mathrm{sw}}$ mentioned earlier and pointed out in FIG. 7(b) by the dotted black line (see details in supplemental material). Nevertheless, as an intermediate conclusion for practical application one can state that a way to reduce the write power consumption is to design the MTJ stack to be close to the condition $K+2 K_{2}=0$. However other parameters must be taken into account as explained in the following.
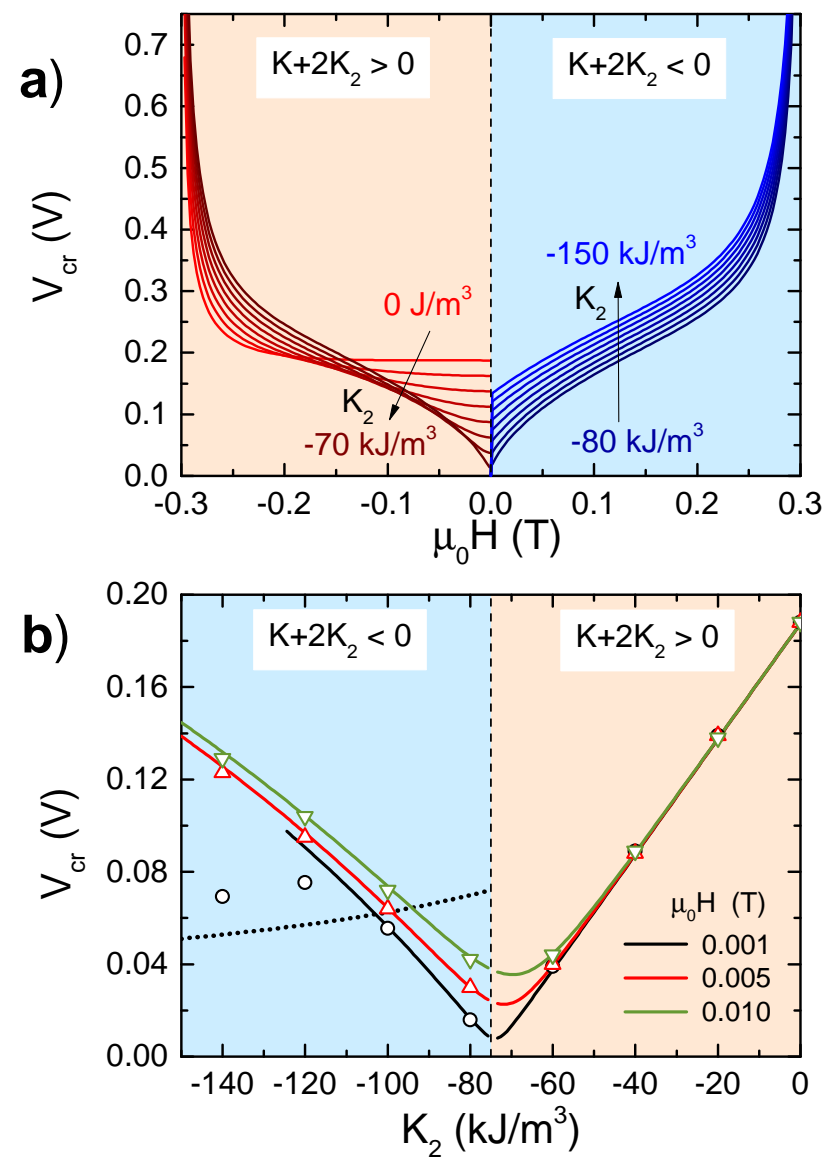

FIG. 7. (a) Critical voltage dependence versus in-plane applied field for several values of $K_{2}$ varying by step of $10 \mathrm{~kJ} / \mathrm{m}^{3}$. Parameters are the same as in FIG. 6. (b) Critical voltage dependence versus $K_{2}$ for several values of in-plane applied field. The colored curves are given by relation (17). The black curve for $\mu_{0} H=1 \mathrm{mT}$ ends up at the limit defined by expression (12). The circles and triangles are the numerical values from full LLG integration for different in-plane fields. The dotted line shows the switching voltage $V_{\mathrm{sw}, 0}$ from the expression (19).

From practical point of view one parameter of importance often considered as the figure of merit in STTMRAM is the ratio between the thermal stability and the switching current. This ratio is plotted in FIG. 8a as predicted analytically from Eq. (17) in the case of an infinite voltage pulse (DC). We supposed that the MTJ has a TMR of $100 \%$ varying between $5 \mathrm{k} \Omega$ and $2.5 \mathrm{k} \Omega$ and the temperature was set at $300 \mathrm{~K}$. The analytical model predicts a large increase of the ratio in the vicinity of the $K_{2}$ threshold. However, the numerical results extracted from the full LLG integration at $300 \mathrm{~K}$ and averaged over 500 measurements show a peak less pronounced in FIG. 8b. Moreover upon reducing the pulse duration the maximum of the curve is disappearing, the curve becomes flatter and a change of regime is observed for pulses below $10 \mathrm{~ns}$. 


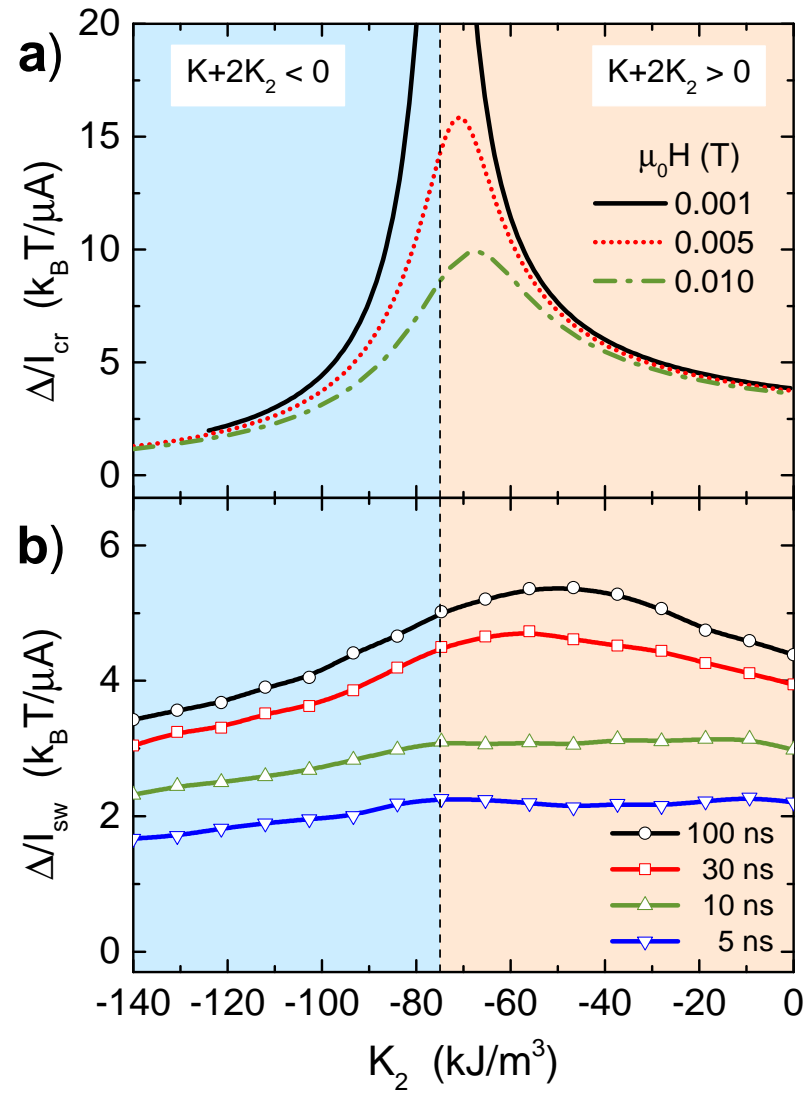

FIG. 8. (a) Energy barrier over switching current calculated from analytics in the limit of small in-plane applied field at $0 \mathrm{~K}$ and static DC voltage for variable $K_{2}$. (b) The same ratio estimated numerically at $300 \mathrm{~K}$ for different pulse durations, no applied field and averaged upon 500 events. Parameters are the same as in FIG. 6 and the free layer has a volume $\Omega=1.96 \cdot 10^{3} \mathrm{~nm}^{3}$.

The explanation of this behavior is related to the switching distribution evolution with the $K_{2}$ strength. Upon reinforcing $K_{2}$, the switching voltage distributions progressively move towards smaller values and their width becomes narrower as depicted in FIG. 9. In the inset of FIG. 9, the switching voltage variation with the pulse duration is shown. As one can see, there are two regimes: above $30 \mathrm{~ns}$, the voltage is almost constant while a sharp increase is observed for very short pulses below 5 ns.

The pulse duration for STT memory application such as embedded-FLASH replacement is in the range of $10 \mathrm{~ns}$. Taking as a reference this pulse duration, the switching voltage dependence on $K_{2}$ for two different temperatures $300 \mathrm{~K}$ and $30 \mathrm{~K}$ is represented in FIG. 10. In the easy-axis regime, the thermal fluctuations are mandatory for the initiation of the magnetization switching. Thus the two curves are clearly separated. In contrast, in the easy-cone

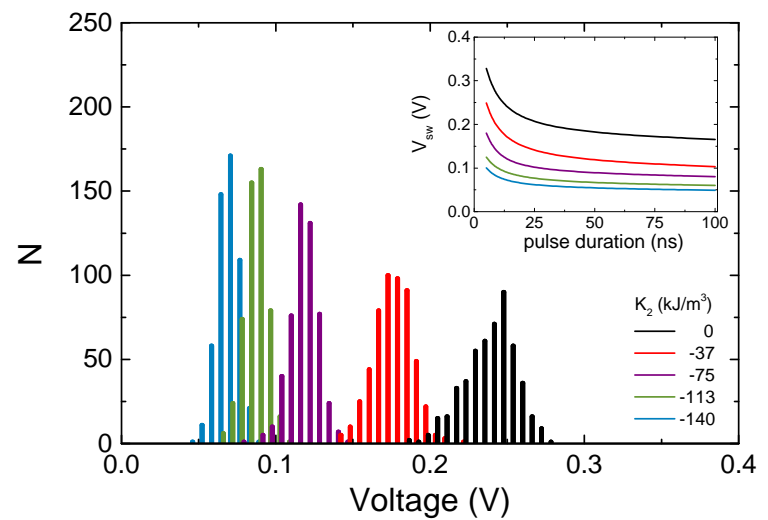

FIG. 9. Number of switching operations versus voltage pulse amplitude after 500 loops at $T=300 \mathrm{~K}$ for a pulse length of $10 \mathrm{~ns}$ for different values of $K_{2}$. Inset: dependence of switching voltage versus writing pulse duration for the same $K_{2}$ values.

regime, the switching voltage is independent of temperature. In addition, due to the intrinsic tilt of the magnetization, the two curves are superposed.

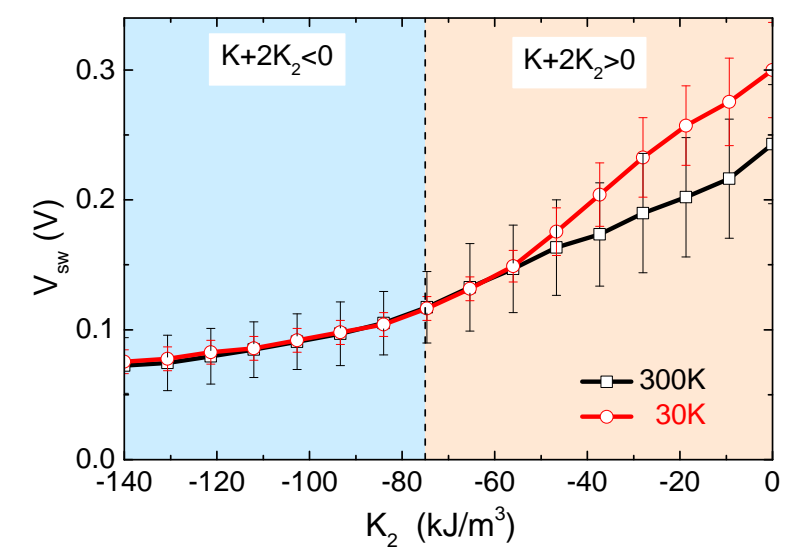

FIG. 10. Switching voltage versus $K_{2}$ for $T=300 \mathrm{~K}$ and $T=30 \mathrm{~K}$ for a pulse duration of $10 \mathrm{~ns}$, the vertical bars are the width of the voltage distribution, Parameters are the same as in FIG. 8 b.

The easy-cone state is thus favorable for memory applications since the writing stochasticity is considerably reduced. However, the $K_{2}$ affects not only the switching voltage but also the energy barrier as given by the following relation:

$$
\frac{E_{B}}{\Omega}=\left\{\begin{array}{ll}
\frac{K^{2}}{4\left|K_{2}\right|}, & K+2 K_{2}<0 \\
K+2 K_{2}, & K+2 K_{2}>0
\end{array} .\right.
$$

The energy barrier over $k_{B} T$ is shown in FIG. 11a by the grey line. The stability factor is continuously 
decreasing upon reinforcing $K_{2}$. At the onset of the easy-cone regime $\left(K+2 K_{2}=0\right)$, the stability factor $\Delta=E_{B} /\left(k_{B} T\right)$ has decreased already by $50 \%$ with respect to the situation $K_{2}=0$. This might be seen as a detrimental factor for memory application. Nevertheless, the energy consumption of the memory (left axis FIG. 11a) is also continuously decreasing approaching tens of fJ for 5 ns pulses.

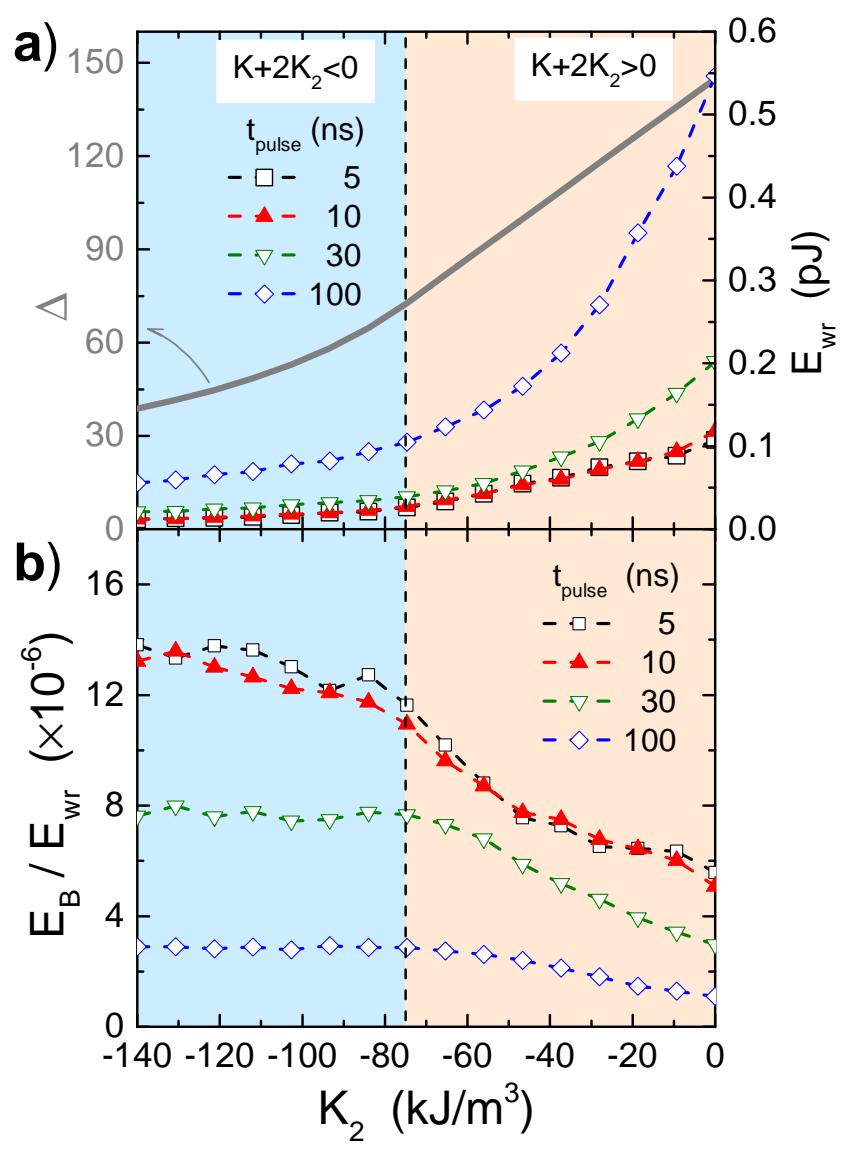

FIG. 11. (a) Dependence of $\Delta(T=300 \mathrm{~K})$ and writing energy on the constant $K_{2}$ for several pulse durations. (b) Ratio $E_{B} / E_{\mathrm{wr}}$ for different pulse durations. Parameters are the same as in FIG. 6.

The ratio $E_{B} / E_{\mathrm{wr}}$ is reported in FIG. $11 \mathrm{~b}$. This ratio can be considered as another figure of merit since it characterizes the tradeoff between thermal stability of the storage layer magnetization and the energy to switch it, which depends of the write current but also on the pulse duration. FIG. 11b shows a significant increase of this ratio for short pulse duration for increasingly large negative $K_{2}$ values. This increase is observed over the whole range of $K_{2}$ values. In the easy-cone regime, this is again due to the reduced stochasticity of the switching due to the pre-existing initial angle between the storage layer magnetization and the spin current polarization which allows to trigger the switching immediately after the on- set of the write pulse. But even in the easy axis regime, when $K+2 K_{2}>0$, the presence of the second order anisotropy has a beneficial influence. This is due to the flatter shape of the energy potential around the direction normal to the plane of the layer. Because of this flatter shape, weaker thermal fluctuations can produce larger angle fluctuations of the storage layer magnetization thus reducing the incubation time preceding the storage layer magnetization reversal.

As a result, second order anisotropy term appears to be quite beneficial for fast STT-MRAM even in the easyaxis regime as long as the thermal stability of the storage layer magnetization can be maintained sufficiently large to cope with the memory retention specification.

\section{CONCLUSIONS}

We performed a systematic experimental investigation of MTJ in non-collinear geometry. The experimental voltage-field stability diagrams exhibit a strong dependence on the direction of the external field with respect to the normal to the layers. By employing numerical modelling based on the LLG integration, we showed that an additional second order uniaxial anisotropy term of the form $-K_{2} \cos ^{4} \theta$ should be taken into account to explain the experimental observations. Despite the fact that the application of non-zero in-plane magnetic field $H_{x}$ introduces an initial non-collinearity, we have not found any noticeable decrease of the threshold current with increasing of $\left|H_{x}\right|$. This is mainly due to the fact that the non-collinearity has two opposite impacts. On one hand, it helps the initiation of the STT driven dynamics. On the other hand, it decreases the efficiency of the STT torque absorption since the precession orbit axis becomes tilted with respect to the polarizer due to the influence of the non-zero in-plane field $H_{x}$. This conclusion is fully confirmed by macrospin simulations. Furthermore, an analytical model for a critical line $V_{\mathrm{cr}}(H)$ in field-in-plane geometry was developed by linearization of the LLG equation allowing to straightforwardly characterize the free layer state. For memory applications the second order anisotropy appears to be an interesting parameter to control and adjust, since it allows to write with lower energy and less stochastically while preserving a reasonable thermal stability.

Acknowledgements: The work was partially supported by ERC Adv grant MAGICAL n ${ }^{\circ} 669204$ and French National Research Agency (ANR) under project EXCALYB. 


\section{APPENDIX}

Coefficients of linearized LLG system of equations:

$$
\begin{aligned}
& A_{0}=\frac{\gamma}{\mu_{0}\left(1+\alpha^{2}\right)}\left(\mu_{0} H_{x}\left(\alpha \cos \theta_{0} \cos \varphi_{0}-\sin \varphi_{0}\right)\right. \\
& \left.\left.-\sin \theta_{0} \frac{2 \alpha \cos \theta_{0}}{M_{s}}\left(K+2 K_{2} \cos ^{2} \theta_{0}\right)+a_{\|} V\right)\right) \\
& A_{1}=-\frac{\gamma H_{x}}{1+\alpha^{2}}\left(\alpha \cos \theta_{0} \sin \varphi_{0}+\cos \varphi_{0}\right) \\
& A_{2}=-\frac{\gamma}{\mu_{0}\left(1+\alpha^{2}\right)}\left(\alpha \mu_{0} H_{x} \sin \theta_{0} \cos \varphi_{0}+\frac{2 \alpha}{M_{s}}\right. \\
& \left.\times\left(\left(K+K_{2}\right) \cos 2 \theta_{0}+K_{2} \cos 4 \theta_{0}\right)+a_{\|} V \cos \theta_{0}\right) \\
& B_{0}=-\frac{\gamma}{\mu_{0}\left(1+\alpha^{2}\right)}\left(-\frac{2 \cos \theta_{0}}{M_{s}}\left(K+2 K_{2} \cos ^{2} \theta_{0}\right)\right. \\
& \left.+\frac{\mu_{0} H_{x}}{\sin \theta_{0}}\left(\cos \theta_{0} \cos \varphi_{0}+\alpha \sin \varphi_{0}\right)+\alpha a_{\|} V\right) \\
& B_{1}=-\frac{\gamma H_{x}}{\left(1+\alpha^{2}\right) \sin \theta_{0}}\left(\alpha \cos \varphi_{0}-\cos \theta_{0} \sin \varphi_{0}\right) \\
& B_{2}=\frac{\gamma}{\mu_{0}\left(1+\alpha^{2}\right)}\left(-\frac{2 \sin \theta_{0}}{M_{s}}\left(K+6 K_{2} \cos ^{2} \theta_{0}\right)\right. \\
& \left.+\frac{\mu_{0} H_{x}}{\sin ^{2} \theta_{0}}\left(\cos \varphi_{0}+\alpha \cos \theta_{0} \sin \varphi_{0}\right)\right)
\end{aligned}
$$

*nik@magn.phys.msu.ru

$\dagger$ bernard.dieny@cea.fr

[1] S. Monso, B. Rodmacq, S. Auffret, G. Casali, F. Fettar, B. Gilles, B. Dieny, and P. Boyer, Applied Physics Letters 80, 4157 (2002).

[2] B. Rodmacq, S. Auffret, B. Dieny, S. Monso, and P. Boyer, Journal of Applied Physics 93, 7513 (2003).

[3] A. Manchon, C. Ducruet, L. Lombard, S. Auffret, B. Rodmacq, B. Dieny, S. Pizzini, J. Vogel, V. Uhlír, M. Hochstrasser, and G. Panaccione, Journal of Applied Physics 104, 043914 (2008).

[4] B. Rodmacq, A. Manchon, C. Ducruet, S. Auffret, and B. Dieny, Phys. Rev. B 79, 024423 (2009).

[5] S. Ikeda, K. Miura, H. Yamamoto, K. Mizunuma, H. D. Gan, M. Endo, S. Kanai, J. Hayakawa, F. Matsukura, and H. Ohno, Nat Mater 9, 721 (2010).
[6] L. E. Nistor, B. Rodmacq, S. Auffret, A. Schuhl, M. Chshiev, and B. Dieny, Phys. Rev. B 81, 220407 (2010).

[7] S. Mangin, Y. Henry, D. Ravelosona, J. A. Katine, and E. E. Fullerton, Applied Physics Letters 94, 012502 (2009).

[8] H. Sato, E. C. I. Enobio, M. Yamanouchi, S. Ikeda, S. Fukami, S. Kanai, F. Matsukura, and H. Ohno, Applied Physics Letters 105, 062403 (2014).

[9] J. J. Nowak, R. P. Robertazzi, J. Z. Sun, G. Hu, J. H. Park, J. Lee, A. J. Annunziata, G. P. Lauer, R. Kothandaraman, E. J. O'Sullivan, P. L. Trouilloud, Y. Kim, and D. C. Worledge, IEEE Magnetics Letters 7, 1 (2016).

[10] J. Slonczewski, Journal of Magnetism and Magnetic Materials 159, L1 (1996).

[11] L. Berger, Phys. Rev. B 54, 9353 (1996).

[12] D. E. Nikonov, G. I. Bourianoff, G. Rowlands, and I. N. Krivorotov, Journal of Applied Physics 107, 113910 (2010).

[13] O. Redon, B. Dieny, and B. Rodmacq, "Magnetic spin polarization and magnetization rotation device with memory and writing process, using such a device" (2003), US Patent 6532164.

[14] A. D. Kent, B. Özyilmaz, and E. del Barco, Applied Physics Letters 84, 3897 (2004).

[15] C. Papusoi, B. Delaët, B. Rodmacq, D. Houssameddine, J.-P. Michel, U. Ebels, R. C. Sousa, L. Buda-Prejbeanu, and B. Dieny, Applied Physics Letters 95, 072506 (2009).

[16] N. N. Mojumder and K. Roy, IEEE Transactions on Electron Devices 59, 3054 (2012).

[17] D. Apalkov, "Method and system for providing magnetic tunneling junction elements having a biaxial anisotropy" (2013), US Patent 8374048.

[18] A. A. Timopheev, R. Sousa, M. Chshiev, H. T. Nguyen, and B. Dieny, Scientific Reports 6, 26877 (2016).

[19] B. Dieny and A. Vedyayev, Europhys. Lett. 25, 723 (1994).

[20] A. A. Timopheev, R. Sousa, M. Chshiev, L. D. BudaPrejbeanu, and B. Dieny, Phys. Rev. B 92, 104430 (2015).

[21] L. Cuchet, B. Rodmacq, S. Auffret, R. C. Sousa, I. L. Prejbeanu, and B. Dieny, Scientific Reports 6, 21246 (2016).

[22] J. M. Shaw, H. T. Nembach, M. Weiler, T. J. Silva, M. Schoen, J. Z. Sun, and D. C. Worledge, IEEE Magnetics Letters 6, 1 (2015).

[23] J. Z. Sun, Phys. Rev. B 91, 174429 (2015).

[24] R. Matsumoto, H. Arai, S. Yuasa, and H. Imamura, Applied Physics Express 8, 063007 (2015).

[25] M. Lavanant, S. P. Watelot, A. Kent, and S. Mangin, JMMM (2016), in press.

[26] K. Bernert, V. Sluka, C. Fowley, J. Lindner, J. Fassbender, and A. M. Deac, Phys. Rev. B 89, 134415 (2014).

[27] S. Yan, Z. Sun, and Y. B. Bazaliy, Phys. Rev. B 88, 054408 (2013).

[28] L. D. Landau and E. M. Lifshitz, Phys. Z. Sowjet. 8, 153 (1935).

[29] T. L. Gilbert, IEEE Transactions on Magnetics 40, 3443 (2004).

[30] S.-C. Oh, S.-Y. Park, A. Manchon, M. Chshiev, J.-H. Han, H.-W. Lee, J.-E. Lee, K.-T. Nam, Y. Jo, Y.-C. Kong, B. Dieny, and K.-J. Lee, Nat Phys 5, 898 (2009). 
[31] M. Chshiev, A. Manchon, A. Kalitsov, N. Ryzhanova, A. Vedyayev, N. Strelkov, W. H. Butler, and B. Dieny, Phys. Rev. B 92, 104422 (2015).

[32] M. Wilczyński, J. Barnaś, and R. Świrkowicz, Phys. Rev. B 77, 054434 (2008).

[33] J. Xiao, G. E. W. Bauer, and A. Brataas, Phys. Rev. B 77, 224419 (2008).

[34] C. Heiliger and M. D. Stiles, Phys. Rev. Lett. 100, 186805 (2008).

[35] A. Manchon, N. Ryzhanova, A. Vedyayev, M. Chschiev, and B. Dieny, Journal of Physics: Condensed Matter 20, 145208 (2008).

[36] M. Chshiev, I. Theodonis, A. Kalitsov, N. Kioussis, and W. H. Butler, IEEE Transactions on Magnetics 44, 2543 (2008).
[37] A. Kalitsov, W. Silvestre, M. Chshiev, and J. P. Velev, Phys. Rev. B 88, 104430 (2013).

[38] A. Manchon, S. Zhang, and K.-J. Lee, Phys. Rev. B 82, 174420 (2010).

[39] B. Schulz and K. Baberschke, Phys. Rev. B 50, 13467 (1994).

[40] M. Farle, B. Mirwald-Schulz, A. N. Anisimov, W. Platow, and K. Baberschke, Phys. Rev. B 55, 3708 (1997).

[41] W. F. Brown, Phys. Rev. 130, 1677 (1963).

[42] J. L. García-Palacios and F. J. Lázaro, Phys. Rev. B 58, 14937 (1998).

[43] P.-H. Jang, S.-W. Lee, and K.-J. Lee, Current Applied Physics 16, 1550 (2016). 